\title{
Non-equilibrium Dynamical Processes in the Galaxy
}

\author{
Alice. C Quillen and Ivan Minchev \\ Dept. of Physics and Astronomy, University of Rochester, \\ Rochester, NY 14627, USA \\ Université de Strasbourg, CNRS, Observatoire Astronomique \\ 11 Rue de l'Université, 67000 Strasbourg, FRANCE
}

\begin{abstract}
Dynamical models have often necessarily assumed that the Galaxy is nearly steady state or dynamically relaxed. However observed structure in the stellar metallicity, spatial and velocity distributions imply that heating, mixing and radial migration has taken place. Better comprehension of non-equilibrium processes will allow us to not only better understand the current structure of the galaxy but its past evolution.
\end{abstract}

During a Hubble time the Milky Way disk at the radius of the Sun has only had time to rotate 40 or 50 times. There is little time for dynamical relaxation. As larger and more precise surveys are conducted we expect even more structure to be revealed in the stellar abundance and phase space distributions. As there is little time for relaxation, structure in the phase space distribution depends on the evolution of the Galaxy.

Resonances with a bar or spiral structure cause stars to move in non-circular orbits. Libration times in Lindblad resonances can be long so evolution could take place in the non-adiabatic limit. Minchev et al. (2009b) proposed that the division between the Pleiades and moving groups in the solar neighborhood is associated with librations in the 2:1 Lindblad resonance with the Galactic bar (see Figure 1). These oscillations are also seen as long lived R1/R2 asymmetric ring structures in test particle simulations of bar growth (Bagley et al. 2009).

If pattern speeds vary, then either particles are trapped into resonance or heated as they cross the resonance. When particles are trapped into resonance their eccentricity depends on the total pattern speed change after capture. When particles cross the resonance their eccentricity can be predicted from the resonance strength and order. Bagley et al. (2009) suggested that the morphology of ring structures associated with a bar depends on bar pattern speed variations since growth. Peanut shaped bulges can also be modeled with a resonant trapping model (Quillen 2002). When there is more than one perturbation, chaotic heating occurs in resonances (Quillen 2003, Minchev \& Quillen 2006).

Since resonances are often narrow, they can be used to place tight constraints on their pattern speed. Their location in the galaxy could be used to measure the pattern speed of a distant spiral pattern with a deep radial velocity survey (Minchev \& Quillen 2007). Resonances occur when the sum of integer multiple of a star's orbital frequencies is equal to an integer multiple of a perturbing frequency such as a planet's mean motion or the pattern speed of a bar. In the Galaxy the orbital period is estimated from the tangential or $v$ velocity component at a particular location. Thus resonances can be located on a $u, v$ plane velocity distribution. Divisions between streams can be used to estimate bar or spiral pattern speeds (Dehnen 1999, Quillen \& Minchev 2005). By matching both the velocity distribution in the solar neighborhood and simulated Oort 


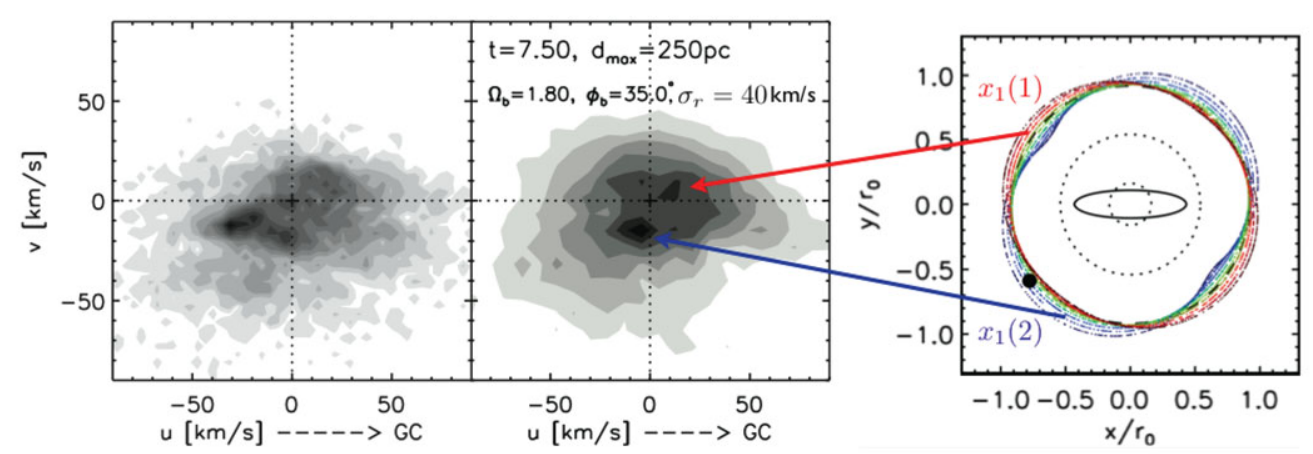

Figure 1. Orbits associated with the Bar. On the left we show the Hipparcos stellar velocity distribution (Dehnen 1998). The middle panel shows a model distribution by Minchev et al. (2009b). On the right we show orbits associated with the Sirius and Pleiades moving groups.

function measurements (that depend on velocity gradients) Minchev et al. (2007) placed an even tighter constraint on the bar pattern speed.

Differences between orbital frequencies cause the velocity and spatial distribution of stars in a narrow region in phase space to spread. This process is called "phase wrapping" and can be used to estimate the time since a merger occurred (Gomez \& Helmi 2009). Uneven distributions in phase space could also be caused by large scale perturbations to the disk. The timescale for them to wrap places constraints on the time since perturbation (Minchev et al. 2009). These scenarios are proposed explanations for high velocity streams in the thick disk of the Galaxy. Both mergers (Quillen et al. 2009) and resonances (Sellwood \& Binney 2002) cause radial migration. Future work can better explore the relation between structure in the phase space and abundance distributions.

In summary, dynamical structures and events leave signatures in the stellar distributions. Precise measurements can be made as observations and associated models become more comprehensive. Unveiling the current and past structure and evolution of the Galaxy will be increasingly exciting in the coming decade.

\section{References}

Bagley, M., Minchev, I., \& Quillen, A. C. 2009, MNRAS, 395, 537

Dehnen, W. 1998, AJ, 115, 2384

Dehnen, W. 1999, ApJ, 524, L34

Gomez, F. \& Helmi, A., MNRASin press, 2009, arXiv0904.1377

Minchev, I. \& Quillen, A. C. 2008, MNRAS, 386, 1579

Minchev, I. \& Quillen, A. C. 2006, MNRAS, 368, 623

Minchev, I., Boily, C., Siebert, A., \& Bienayme, O. 2009, submitted to MNRAS, arXiv0909.3516

Minchev, I., Quillen, A. C., Williams, M., Freeman, K. C., Nordhaus, J., Siebert, A., \& Bienaymé, O. 2009, MNRAS, 396, L56

Minchev, I., Nordhaus, J., \& Quillen, A. C. 2007, ApJ, 664, L31

Quillen, A. C., Minchev, I., Bland-Hawthorn, J., \& Haywood, M. 2009, MNRAS, 397, 1599

Quillen, A. C., \& Minchev, I. 2005, AJ, 130, 576

Quillen, A. C. 2003, AJ, 125, 785

Quillen, A. C. 2002, $A J, 124,722$

Sellwood, J. A. \& Binney, J. J. 2002, MNRAS, 336, 785 